\title{
The Impact of Retroactivity on Information Exchange in Molecular Communications
}

\author{
Francesca Ratti \\ Massachusetts Institute of Technology \\ Cambridge, Massachusetts, USA \\ Politecnico di Milano \\ Milan, Italy \\ fratti@mit.edu
}

\author{
Maurizio Magarini \\ Politecnico di Milano \\ Milan, Italy \\ maurizio.magarini@polimi.it
}

\author{
Domitilla Del Vecchio \\ Massachusetts Institute of Technology \\ Cambridge, Massachusetts, USA \\ ddv@mit.edu
}

\begin{abstract}
Information exchange is a key process in all communication systems, including biological ones. This paper presents first results on the study of the impact of retroactivity, the loads that downstream modules apply on their upstream systems, on different biological signaling system models. The aim is to provide analytical tools to maximize the reliable information exchange in biomolecular circuits.
\end{abstract}

\section{ACM Reference Format:}

Francesca Ratti, Maurizio Magarini, and Domitilla Del Vecchio. 2020. The Impact of Retroactivity on Information Exchange in Molecular Communications. In The Seventh Annual ACM International Conference on Nanoscale Computing and Communication (NANOCOM '20), September 23-25, 2020, Virtual Event, USA. ACM, New York, NY, USA, 2 pages. https://doi.org/10. $1145 / 3411295.3411311$

\section{INTRODUCTION}

Molecular Communication (MC) is a field of study that is earning relevance in recent years [1]. There exist several works investigating parallelisms between well-known telecommunication and MC models - e.g. broadcast channel and multiple input multiple output (MIMO) channel - and evaluating information exchange performances in different biological scenarios [2, 3].

Systems biology is a discipline that studies natural systems [4], while synthetic biology studies how to engineer them [5]. Both these two subjects are other promising fields of study. Among the concepts that have been explored in these areas, a key phenomenon is that of retroactivity. Retroactivity, not to be confused with feedback, is the back action from a downstream system connected to an upstream one, which is observed in biomolecular systems [4]. A review of retroactivity for different signaling systems and genetic circuits can be found in [6].

We aim to investigate the impact of the retroactivity on the information exchange for different $\mathrm{MC}$ system models. The ultimate objective is to lay foundations of how to maximize the reliable information exchange in different scenarios. In the following, we illustrate preliminary results we achieved in this direction.

This work is licensed under a Creative Commons Attribution International 4.0 License. NANOCOM '20, September 23-25, 2020, Virtual Event, USA

(C) 2020 Copyright held by the owner/author(s)

ACM ISBN 978-1-4503-8083-6/20/09.

https://doi.org/10.1145/3411295.3411311

\section{RETROACTIVITY AND INFORMATION EXCHANGE PERFORMANCE}

In the following, we will consider different MC models, which recall in some aspects well-known telecommunication systems. The common thread in the different models is the presence of an input protein I, i.e. the input message, with a probability mass function (pmf) on the transmitted symbols injected in the MC system at time $t_{0} P\left(\mathrm{I}_{t_{0}}\right)$ and of a destination message, a protein $\mathrm{Z}$. Each transmitted symbol is composed by a number of molecules of I. A chain of chemical reactions leads to the production of $\mathrm{Z}$ starting from I. The number of molecules of the output protein $Z$ present in the MC system is evaluated at steady-state, to avoid the effects of the transient behavior of the chemical reactions. We refer to the pmf of the destination protein $\mathrm{Z}$ at steady-state as $P\left(\mathrm{Z}_{t_{s}}\right)$.

We quantify the amount of information exchanged between input and output message with the mutual information (MI) defined as difference of entropies [7] $I\left(\mathrm{I}_{t_{0}}, \mathrm{Z}_{t_{s}}\right)=H\left(\mathrm{I}_{t_{0}}\right)+H\left(\mathrm{Z}_{t_{s}}\right)-$ $H\left(\mathrm{I}_{t_{0}}, \mathrm{Z}_{t_{s}}\right)$, where $H\left(\mathrm{I}_{t_{0}}\right), H\left(\mathrm{Z}_{t_{s}}\right), H\left(\mathrm{I}_{t_{0}}, \mathrm{Z}_{t_{s}}\right)$ are, respectively, the entropies of $I$ at time $t_{0}$, of $Z$ at steady-state and the joint entropy of $\mathrm{I}_{t_{0}}$ and $\mathrm{Z}_{t_{s}}$. Notice that we consider $\mathrm{I}$ and $\mathrm{Z}$ at two different instants of time. This is because we want to examine the information exchange between the input message at the time in which transmission starts, that is $t_{0}$, and the destination message at the time it is fully received, i.e at steady-state.

The entropy of a generic discrete variable $X$ depends on its pmf $P(X)$ as $H(X)=-\sum_{i=0}^{N} P\left(x_{i}\right) \log _{b} P\left(x_{i}\right)$ [7], where $b$ is the base of the logarithm that determines its unit of measure. Therefore, we need to evaluate $P\left(\mathrm{I}_{t_{0}}\right), P\left(\mathrm{Z}_{t_{s}}\right)$ and $P\left(\mathrm{I}_{t_{0}}, \mathrm{Z}_{t_{s}}\right)$ to obtain an analytical form of the information exchange between $I$ and $Z$. The pmf of the input $P\left(\mathrm{I}_{t_{0}}\right)$ is one of the variables on which the MI depends. By solving the Chemical Master Equation (CME) [4] at steady-state, we express $P\left(\mathrm{Z}_{t_{s}}\right)$ and $P\left(\mathrm{I}_{t_{0}}, \mathrm{Z}_{t_{s}}\right)$ as function of both the parameters of the chemical reactions of the system and $P\left(\mathrm{I}_{t_{0}}\right)$. The motivations behind the choice of the CME to model the behavior of the system are two. First, we consider the accuracy of the results worth the complexity of the calculations. Furthermore, the CME does not take into account any stochastic effects other than the ones given by chemical reactions. In this way, we can selectively quantify the effect of retroactivity on information exchange for different biomolecular system models.

In this paper we analyze the three signaling systems [4] showed in Figure 1. The first is an isolated signaling system, which is not connected to any downstream target. The second and the third are connected through $\mathrm{Z}$, respectively, to one and $N$ downstream 

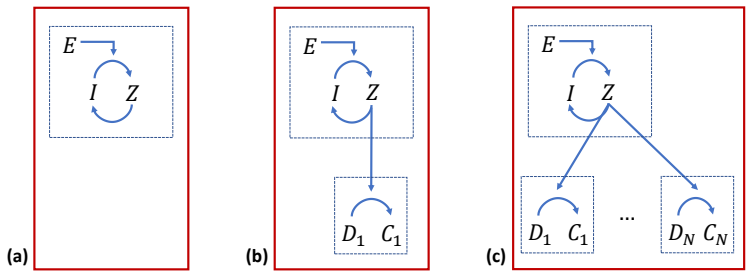

Figure 1: Diagram of the signaling system (a), with one downstream system connected to the output (b), with $N$ downstream systems connected to the output (c).

systems. The third model, having more downstream systems connected, could be seen as a broadcast channel [7], in which the output of the upstream system $\mathrm{Z}$ is the same message sent to different receivers, i.e. the downstream systems. The chemical reactions modeling the upstream system are $\mathrm{I}+\mathrm{E} \stackrel{\mathrm{k}_{D_{0}}}{\rightleftharpoons} \mathrm{M}, \mathrm{M} \stackrel{\mathrm{c}_{1}}{\longrightarrow} \mathrm{E}+\mathrm{Z}$, $\mathrm{Z} \stackrel{\mathrm{C}_{2}}{\longrightarrow}$ I with conservation law $\mathrm{E}_{\text {tot }}=\mathrm{E}+\mathrm{M}$, where $\mathrm{I}$ is the protein (input message) that binds with an enzyme $\mathrm{E}$ to form the complex $M$, that in turn is transformed in the output protein $Z$ [4]. The last chemical reaction composing the upstream system makes it cyclic. This is a major discrepancy with respect to traditional telecommunications models, which are unidirectional. Each $j$ th downstream system is composed of one reversible reaction $\mathrm{Z}+\mathrm{D}_{j} \stackrel{\mathrm{k}_{D_{3_{j}}}}{\rightleftharpoons} \mathrm{C}_{j}$ with conservation law $\mathrm{D}_{t o t}=\mathrm{D}_{j}+\mathrm{C}_{j}$, where the output of the upstream system $\mathrm{Z}$ binds with the DNA D to form the complex C. The coefficients $\mathrm{k}_{D_{0}}$ and $\mathrm{k}_{D_{3 j}}$ are the dissociation constants of the reversible binding reactions and are equal, respectively, to $k_{0}^{-} / k_{0}^{+}$ and $\mathrm{k}_{3_{j}}^{-} / \mathrm{k}_{3_{j}}^{+}$. The last conservation law fulfilled by each of the three considered systems is $\mathrm{I}_{t o t}=\mathrm{I}+\mathrm{M}+\mathrm{Z}+\sum_{j=1}^{N} \mathrm{C}_{j}$. Thus, supposing no $\mathrm{M}, \mathrm{Z}, \mathrm{C}_{j}$ present in the system before $t_{0}$, we can write $\mathrm{I}_{\text {tot }}\left(t_{0}\right)=\mathrm{I}_{t_{0}}$.

\section{RESULTS}

We make hypothesis on the values of $\mathrm{E}_{t o t}, \mathrm{D}_{t o t}$ and on the number of input symbols $n_{I}$ and their corresponding $\mathrm{I}_{t_{0}}$ to compute the solution of the CME. We choose $n_{I}=2$, meaning that there are two available input symbols, one composed by 0 molecules of $\mathrm{I}\left(\mathrm{I}_{t_{0}}=0\right.$, no transmission) while the second composed by one molecule of $\mathrm{I}\left(\mathrm{I}_{t_{0}}=1\right)$. This corresponds to a Concentration On-Off Keying modulation [8]. This choice allows the explicit enumeration of all the microstates of the system, thus the analytical investigation of the role of retroactivity in the information exchange between $\mathrm{I}_{t_{0}}$ and $\mathrm{Z}_{t_{s}}$. We seek to extend our analysis in future work to consider larger molecular counts. Accordingly, we also set $\mathrm{E}_{t o t}, \mathrm{D}_{t o t_{j}}=1$.

It is possible to show that the formula of the MI between $\mathrm{I}_{t_{0}}$ and $\mathrm{Z}_{t_{s}}$ we obtain in the three cases is the same:

$$
\begin{aligned}
& I\left(\mathrm{I}_{t_{0}}, \mathrm{Z}_{t_{s}}\right)=\log _{e}\left(P_{0}^{\left(-P_{0}\right)} P_{1}^{\left(-P_{1}\right)}\right) \\
& -\log _{e}\left(\left(\left(\frac{P_{0}}{P_{0}+A P_{1}}\right)^{\left(-P_{0}\right)}\right) \cdot\left(\left(\frac{A P_{1}}{P_{0}+A P_{1}}\right)^{\left(-A P_{1}\right)}\right)\right)
\end{aligned}
$$

[nat/symbol], where $P_{0}$ and $P_{1}$ are the probabilities of the two possible input symbols and $A$ is a constant depending on the coefficients of the chemical reactions. In the first case, when the signaling system is isolated (Fig. 1(a)), we obtain $A=A_{0}=\frac{\left(1+\mathrm{k}_{D_{0}}\right) \mathrm{c}_{2}}{\mathrm{c}_{1}+\left(1+\mathrm{k}_{D_{0}}\right) \mathrm{c}_{2}}$.
This shows that at steady-state the probability of the output $Z_{t_{s}}$ is affected by the presence of the reaction $\mathrm{Z} \stackrel{\mathrm{c}_{2}}{\longrightarrow}$ I that transforms the output protein $\mathrm{Z}$ in the input I, not allowing the complete consumption of I at steady-state. The term $A$ in the second and third systems (Fig. 1(b), 1(c)) captures the impact of the retroactivity on the information exchange. In these cases, the formula of $A$ is affected by the coefficients of the reaction(s) connecting the upstream and the downstream system(s). In the second case, we obtain $A=A_{1}=1-\frac{\mathrm{c}_{1}}{\left(1+\mathrm{k}_{D_{0}}\right) \mathrm{c}_{2}+\left(1+1 / \mathrm{k}_{D_{3_{1}}}\right) \mathrm{c}_{1}}$ and for the third we have $A=A_{N}=1-\frac{\mathrm{c}_{1}}{\left(1+\mathrm{k}_{D_{0}}\right) \mathrm{c}_{2}+\left(1+\sum_{j=1}^{N}\left(1 / \mathrm{k}_{D_{3_{j}}}\right)\right) \mathrm{c}_{1}}$. If we impose $N=1$ in the formula of $A_{N}$ we obtain $A_{1}$, while if we remove the downstream systems effect $\sum_{j=1}^{N}\left(1 / \mathrm{k}_{D_{3_{j}}}\right)$, we return to $A_{0}$. The last studied system captures the impact of retroactivity on the information exchange performance in a generic biomolecular signaling broadcast channel. If we suppose that all the $N$ downstream systems have the same dissociation constant $\mathrm{k}_{D_{3}}$, we can rewrite $A_{N}=1-\frac{\mathrm{c}_{1}}{\left(1+\mathrm{k}_{D_{0}}\right) \mathrm{c}_{2}+\left(1+N / \mathrm{k}_{D_{3}}\right) \mathrm{c}_{1}}$, that for $N \rightarrow \infty$ is equal to 1 . If we substitute $A=1$ in Eq. (1), we obtain that $I\left(\mathrm{I}_{t_{0}}, \mathrm{Z}_{t_{s}}\right)=0$, i.e. $\mathrm{I}_{t_{0}}, Z_{t_{s}}$ are completely independent. This is a coherent result if we note that, for infinite downstream systems, the probability of the number of free molecules of $Z_{t_{s}}$ being different from 0 tends to 0 , independently from the value of $\mathrm{I}_{t_{0}}$.

We note that for $\mathrm{c}_{2}=0$, i.e. if the upstream system is not cyclic, $A_{0}$ becomes 0 , leading to $I\left(\mathrm{I}_{t_{0}}, \mathrm{Z}_{t_{s}}\right)=\log _{e}\left(P_{0}^{\left(-P_{0}\right)} P_{1}^{\left(-P_{1}\right)}\right)$, i.e. the MI is equal to the entropy of $I_{t_{0}}$. Thus, in this case $I_{t_{0}}$ and $Z_{t_{s}}$ are fully dependent. On the contrary, removing the cycle in the upstream system results in $A_{N}=1-\frac{\mathrm{c}_{1}}{\left(1+\sum_{j=1}^{N}\left(1 / \mathrm{k}_{D_{3_{j}}}\right)\right) \mathrm{c}_{1}}$. This is because the load given by the connected downstream systems still has an impact on the information exchange between I and $\mathrm{Z}$ by subtracting free $Z$ (output message) from the system environment.

\section{CONCLUSION}

In this paper, we show preliminary results on the evaluation of the $\mathrm{MI}$ in different biomolecular signaling circuits. Through the solution of the CME, we obtain an analytical formulation of the MI for different system models and we quantify the impact of retroactivity in different scenarios. This analysis lays the foundation for the maximization of reliable shared information in biological systems.

\section{REFERENCES}

[1] I. F. Akyildiz, F. Brunetti, and C. Blázquez. Nanonetworks: A new communication paradigm. Computer Networks, 52(12):2260-2279, 2008.

[2] Y. Lu, M. D. Higgins, A. Noel, M. S. Leeson, and Y. Chen. The effect of two receivers on broadcast molecular communication systems. IEEE T-NB, 15(8):891-900, 2016.

[3] S. M. Rouzegar and U. Spagnolini. Channel estimation for diffusive mimo molecular communications. In $2017 \mathrm{EuCNC}$, pages 1-5. IEEE, 2017.

[4] D. Del Vecchio and R. M. Murray. Biomolecular feedback systems. Princeton University Press Princeton, NJ, 2015.

[5] S. A. Benner and A. M. Sismour. Synthetic biology. Nature Reviews Genetics, 6(7):533-543, 2005.

[6] C. McBride, R. Shah, and D. Del Vecchio. The effect of loads in molecular communications. Proceedings of the IEEE, 107(7):1369-1386, 2019.

[7] T. M. Cover. Elements of information theory. John Wiley \& Sons, 1999.

[8] M. S. Kuran, H. B. Yilmaz, T. Tugcu, and I. F. Akyildiz. Modulation techniques for communication via diffusion in nanonetworks. In 2011 ICC, pages 1-5. IEEE, 2011. 